This is the accepted version of the manuscript published by Elsevier in Neurobiology of

Learning and Memory, available online: https://doi.org/ 10.1016/j.nlm.2015.02.012

\title{
Nap sleep preserves associative but not item memory performance
}

Sara Studte ${ }^{a}$ (M.Sc.)

Emma Bridger ${ }^{\mathrm{a}}(\mathrm{Dr}$.

Axel Mecklinger ${ }^{\mathrm{a}}$ (Prof. Dr.)

${ }^{\mathrm{a}}$ Experimental Neuropsychology Unit, Saarland University, Germany

e-mail addresses:

s.studte@mx.uni-saarland.de

e.bridger@mx.uni-saarland.de

mecklinger@mx.uni-saarland.de

Corresponding author:

Sara Studte

Experimental Neuropsychology Unit

Post-box 151150

D-66041 Saarbrücken

Tel: 0049-681-302-64365

Fax: 0049-681-302 6516

E-Mail: $\underline{\text { s.studte@mx.uni-saarland.de }}$ 


\section{Abstract}

Many studies have shown that sleep improves memory performance, and that even short naps during the day are beneficial. Certain physiological components of sleep such as spindles and slow-wave-sleep are thought to be particularly important for memory consolidation. The aim of this experiment was to reveal the role of naps for hippocampus-dependent associative memory (AM) and hippocampus-independent item memory (IM) alongside their corresponding ERP old/new effects. Participants learnt single words and word-pairs before performing an IMand an AM-test (baseline). One group was subsequently allowed to nap ( 90 minutes) while the other watched DVDs (control group). Afterwards, both groups performed a final IM- and AM-test for the learned stimuli (posttest). IM performance decreased for both groups, whilst AM performance decreased for the control group but remained constant for the nap group, consistent with predictions concerning the selective impact of napping on hippocampusdependent recognition. Putative ERP correlates of familiarity and recollection were observed in the IM posttest, whereas only the later recollection-related effect was present in the AM test. Notably, none of these effects varied with group. Positive correlations were observed between spindle density during slow-wave-sleep and AM posttest performance as well as between spindle density during non-REM sleep and AM baseline performance, showing that successful learning and retrieval both before and after sleep relates to spindle density during nap sleep. Together, these results speak for a selective beneficial impact of naps on hippocampusdependent memories.

Keywords: Sleep Spindles, Nap, Recognition Memory, Event-Related-Potentials 


\section{INTRODUCTION}

Sleep is thought to play an important role in memory consolidation. An increasing number of studies have shown benefits in different memory tasks after sleep compared to a comparable time awake (Diekelmann \& Born, 2010; Diekelmann, Wilhelm, \& Born, 2009). In declarative memory tasks, sleep benefits have been demonstrated, amongst others, for associated items (Marshall, Molle, Hallschmid, \& Born, 2004; Tucker \& Fishbein, 2008; Tucker et al., 2006) and in spatial memory tasks (Peigneux et al., 2004; Plihal \& Born, 1999). It is currently thought that hippocampus-dependent memory consolidation benefits from nonREM (NREM) sleep, in particular from slow oscillations $(<1 \mathrm{~Hz})$ during slow-wave-sleep (SWS) and associated sleep spindles (oscillations between 12-15 Hz) (Born, Rasch, \& Gais, 2006; Born \& Wilhelm, 2012).

Although there is increasing evidence for the beneficial impact of sleep on declarative memory consolidation, less is known about the impact of nap sleep on recognition memory. The aim of the present study was to use behavioral and ERP measures of recognition memory together with polysomnographic data to investigate the benefits of nap sleep and the mechanisms by which nap sleep enhances declarative memory retention.

To date, a number of studies have shown that the density of sleep spindles is associated with enhanced declarative memory (Gais, Molle, Helms, \& Born, 2002; Mednick et al., 2013; Saletin, Goldstein, \& Walker, 2011; Schabus et al., 2004; Schmidt et al., 2006). Mednick and colleagues (2013) experimentally increased spindle density with a drug during a daytime nap, which led to better word-pair associate memory performance compared with a placebo. A recent study by Cox, Hofman and Talamini (2012) indicated that the beneficial effect of sleep spindles on memory is specific to SWS by showing not only that spindle density in SWS is higher than in light sleep (S2) but that only spindle density in SWS and not in light sleep was positively correlated with memory performance. This pattern suggests that the beneficial effect of sleep 
spindles on memory consolidation is dependent on the co-occurrence of slow oscillations (Cox, Hofman, \& Talamini, 2012).

According to the active system consolidation hypothesis, benefits come about because new declarative information is initially encoded in both the hippocampus and neocortex from where, in a second step, it is gradually transformed so that neocortical memories become independent of the hippocampus (O'Reilly, Bhattacharyya, Howard, \& Ketz, 2011; Rasch \& Born, 2013). It is assumed that much of this transfer takes place during sleep by covert neuronal reactivations (Born \& Wilhelm, 2012; Diekelmann \& Born, 2010). Consistent with this view, neuronal reactivation has been reported during sleep, particularly in regions that were active during encoding (Ji \& Wilson, 2007; Peigneux et al., 2004; Rasch, Buechel, Gais, \& Born, 2007; Sirota, Csicsvari, Buhl, \& Buzsaki, 2003).

Although some of the neurophysiological mechanisms by which sleep can boost declarative memory have been identified, findings of sleep effects on recognition memory are less consistent and much less is known about how recognition memory can benefit from sleep or from nap sleep in particular (Daurat, Terrier, Foret, \& Tiberge, 2007; Drosopoulos, Wagner, \& Born, 2005; Hu, Stylos-Allan, \& Walker, 2006; M. Mograss, Godbout, \& Guillem, 2006; M. A. Mograss, Guillem, \& Godbout, 2008; Wagner, Kashyap, Diekelmann, \& Born, 2007), According to dual process models, recognition memory is composed of two processes (Yonelinas, Aly, Wang, \& Koen, 2010). One is a fast and context-free process, called familiarity and the second, named recollection, is thought to be a slower and effortful process by which contextual details of a prior episode can be recovered (Yonelinas, 2002). These two processes are not mutually exclusive but there is nevertheless evidence that recollection- and familiaritybased recognition decisions are supported by distinct neuronal systems (Skinner, Manios, Fugelsang, \& Fernandes, 2014; Yonelinas, Otten, Shaw, \& Rugg, 2005). In agreement with the general assumption that hippocampus-dependent memory consolidation benefits from sleep, some studies investigating sleep effects on recognition memory demonstrate benefits only for 
recollection but not for familiarity (Daurat et al., 2007; Drosopoulos et al., 2005). Using a word list discrimination task together with a process dissociation procedure to estimate familiarity and recollection, Drosopoulos et al. (2005) found that early and late night sleep enhanced recollection, whereas familiarity was not affected by sleep. Daurat et al. (2007) used a remember/know paradigm to examine the effects of SWS and REM sleep on familiarity and recollection. The recollection estimate was enhanced after a 3-hour retention interval filled with SWS as compared to retention intervals filled with REM sleep or no sleep at all. Once again, familiarity was not modulated by any of the retention interval manipulations.

It remains to be shown, however, whether recollection-specific increases in performance can be induced on the basis of SWS-rich nap sleep alone. This was addressed in the current study using two independent approaches to assess recollection and familiarity. Firstly, two separate recognition tasks - differing in the extent to which recollection is required for task performance - were employed. Secondly, indices of putative neural correlates of recollection and familiarity were recorded.

One kind of memory task which is thought to make familiarity-based decisions insufficient to support correct responding are associative tests (Yonelinas et al., 2010). Whereas in item memory (IM) tests stimuli can be either classified as old (learnt) or new (not learnt) on the basis of familiarity as well as recollection, in associative memory (AM) tests subjects are required to discriminate between old (learnt) pairs and recombined (learnt but new configurations of items) pairs. By this, associative memory tests provide a more sensitive measure for recollection than item memory tests, because old and recombined pairs cannot be discriminated on the basis of familiarity (Hockley \& Consoli, 1999; Yonelinas, 1997) ${ }^{1}$ and finding sleep related changes in an associative memory task and no corresponding differences in an item memory task can be taken as evidence that recollection is principally affected by nap

${ }^{1}$ Under some circumstances familiarity is thought to be useful in associative tests, i.e. with certain kinds of semantic associations (Kriukova, Bridger, \& Mecklinger, 2013). The current study used unrelated word-pairs to minimize this. 
sleep.. Notably, familiarity- and recollection-based processes have also been associated with distinct event-related potential (ERP) old/new effects (Friedman \& Johnson, 2000; Mecklinger, 2000; Rugg \& Curran, 2007). An early mid-frontal old/new effect has been shown to operate in a way which is consistent with an index of familiarity (Bridger, Bader, \& Mecklinger, 2014) while the late parietal old/new effect has been shown to correlate with recollection-based memory judgments (Curran \& Cleary, 2003) and the amplitude of this late parietal old/new effect varies with the amount recollected (Vilberg, Moosavi, \& Rugg, 2006). To our knowledge, only Mograss et al. (2006, 2008) explored sleep effects on recognition memory using ERPs as a dependent measure so far. They report enhanced performance and larger ERP old/new effects at frontal and posterior recording sites for a sleep as compared to a wake control group. Unfortunately, however, these data were not used to explore the possibility that familiarity and recollection might be differentially impacted by sleep. Polysomnographic data was also not recorded during sleep periods in these studies, precluding the possibility to test for correspondences between enhanced memory performance and specific sleep parameters.

In the present study, the effects of nap sleep on associative and item recognition memory and their reflection in the ERP correlates of familiarity and recollection were examined using a dual process perspective. Based on the aforementioned data points indicating that hippocampus-dependent (declarative) memory seems to benefit from sleep, in particular SWS, we predicted a beneficial effect of sleep on memory performance only in the AM test. This should be reflected by less deterioration from pre- to post-sleep in AM as compared to IM performance for the nap compared to control group. Furthermore, AM posttest performance within the nap group should be associated with high spindle density (in particular spindle density during SWS (Cox et al., 2012)). Corresponding correlations between IM performance and sleep EEG parameters, as well as group differences in IM performance and the ERP correlate of familiarity at posttest were not expected. In line with the expectation that the benefit of hippocampus-dependent memory from sleep reflects an enhancement of recollection, we 
anticipated that the late parietal old/new effect, the putative ERP correlate of recollection, would be larger after sleep compared to the control group.

\section{MATERIALS and METHODS}

\subsection{Participants}

73 healthy young adults from Saarland University/HTW Saarland participated in this experiment. Data from 17 subjects were excluded due to being at chance level in their baseline memory performance (average performance across conditions at or below $50 \%$ in the IM baseline test and/or $33 \%$ in the AM baseline test). The remaining 56 participants were randomly divided into two groups, either a nap or a control group. Data from an additional 15 subjects were excluded due to performance below 2 SD of the mean of the group at IM posttest and/or AM posttest $(n=5)$, not sleeping (no occurrence of stage 2 sleep) in the nap group $(n=5)$, or sleeping (occurrence of stage 2 sleep) while being in the wake control group $(n=5)$. From the remaining 41 participants, the nap group $(n=22)$ consisted of 13 females and 9 males with a mean age of 22.1 (SD 2.4). The mean age of the control group ( $n=19,10$ females) was 22.1 (SD 2.2) years. All participants stated that they did not have any sleep disorders, had no known neurological problems and that they were right-handed (Oldfield, 1971). All participants gave written informed consent and were paid at a rate of $8 € / \mathrm{h}$ or with course credit.

\subsection{Stimuli}

180 German nouns (Bridger \& Mecklinger, 2012) and 180 semantically unrelated German word-pairs were used as stimuli. All single words were concrete nouns with a length between 3-9 letters and a frequency between 6 and 869 (Baayen, Piepenbrock, \& Gulikers, 1995). 168 of the word-pairs were evaluated in terms of semantic relationship and suitability to 
build a compound in order to reduce the pre-experimental associations within pairs (Bader, Mecklinger, Hoppstadter, \& Meyer, 2010) and 12 were newly created using the same evaluation criteria. For all 180 word-pairs recombined pairs were created using the same evaluation criteria as for the new pairs. To build recombined word-pairs, study pairs were separated into two different lists within each block, each of which corresponded to items to be presented either within the baseline test or posttest. Within blocks, single words were recombined but the position of words (first or second within a pair) remained constant across study and test. An additional 30 subjects rated the semantic relatedness and unitizability of the new and recombined word-pairs and only word-pairs with low semantic relatedness and low unitization values (each $\leq 2$ on a scale from 1-4) were included as test stimuli. All word-pairs had a mean length of 4-8 letters and a mean frequency between 6.5 and 454.5 . The order of learning and testing single words or word-pairs first was counterbalanced across participants. In total, there were six different stimuli-sets for single words and nine different stimuli-sets for word-pairs which ensured that, across our sample, all items appeared equally often as old or new (IM) or as old/new/recombined (AM).

\subsection{Design and Procedure}

The experiment was divided into two sessions (see Fig. 1) which were separated by at least 7 days. The first session served to record various covariate measures e.g. IQ (CFT 20-R) and to explain the sleep log which was to be filled in for one week prior to session 2 . The sleep $\log$ asked for habitual bed, wake and rise times as well as for the occurrence of day naps and the ingestion of alcohol. Feeling of tiredness was also measured over several time points during the day. Participants were instructed to maintain a normal sleep/wake pattern during the week but were asked to sleep one hour less than their average from day 6 to day 7 (experimental day 2) if possible, to increase their sleep pressure. 
Session 2 always started at $13.45 \mathrm{pm}$ with the electrode setup (see below) and the Epworth and Stanford Sleepiness Scales. The Epworth Sleepiness Scale is a questionnaire measuring daily sleepiness by assessing the likelihood of falling asleep in different situations. The Stanford Sleepiness Scale (SSS) measures the current feeling of sleepiness ranging on a 17 scale. Both groups were asked about their feeling of sleepiness at 4 different time points, SSS1: before learning; SSS2: after baseline test; SSS3: after watching the DVD; and SSS4: at the end of the experiment. The nap group was additionally measured at an extra time point (SSS3a) after waking from their nap. Two electrodes were applied to the chin of participants in the nap group to measure muscle activity during sleep, before these participants were asked to lie down at around 15:30 pm ( \pm 15 minutes). Participants were given the opportunity to sleep for a maximum of 90 minutes (see Fig 1). The control group watched two movies: Powaqqatsi and Relaxing: The most beautiful landscapes on earth. Both are movies with only instrumental sound, lasting in sum 2 hours. After waking, nap participants also watched 30 minutes of the Relaxing movie to prevent any sleepiness effects on the second test (posttest). This also ensured that the interval between baseline and posttest was matched for the two groups.

- Insert Figure 1 around here -

\section{Memory tasks}

The memory tasks were programmed using E-Prime 2.0 (Psychology Software Tools, E-Studio 2.0.8.90). Participants sat in front of the monitor at a viewing distance of about $65 \mathrm{~cm}$. Stimuli were presented in black on a grey background (maximal horizontal visual angle $\approx 5.7^{\circ}$ ). Single words were presented in the center of the screen (vertical visual angle $\approx 1.3^{\circ}$ ), whereas word-pairs were presented slightly below and above central vision in study and test phases (vertical visual angle $\approx 4^{\circ}$ ). The learning of single words and word-pairs was blocked and whether participants first learnt single words or word-pairs was counterbalanced. The presentation time of all stimuli at study was $5000 \mathrm{~ms}$. Participants were instructed to memorize 
items for a later memory test but no specific learning strategy was given. The study list of 90 single words was divided into two blocks, while the study list with 120 word-pairs was divided into three blocks. There was a self-paced break in between blocks as well as between the two study-lists. Stimuli were presented in random order with an ISI (fixation cross shown for 500 $\mathrm{ms}$ ) of $550 \mathrm{~ms}$. The duration of the study phase was about 22 minutes.

The first memory test (baseline/pretest) was conducted immediately after the study phase. Here, participants had to decide whether the presented single word was old or new (item memory test, IM) or whether the presented word-pair was old, new or recombined (associative memory test, AM). Participants responded on one of two keys for single words (old/new decision) and on one of three keys for word-pairs (old/new/recombined decision). The key assignment to right and left hand was counterbalanced across subjects. Participants were instructed to respond as fast and as accurately as possible. Single words were presented for 500 ms, followed by a $2000 \mathrm{~ms}$ long response window and an ISI of $1000 \mathrm{~ms}$. Word-pairs were presented for $750 \mathrm{~ms}$, followed by a $2000 \mathrm{~ms}$ long response window with an ISI of $1000 \mathrm{~ms}$. The baseline test included 30 old and 30 new single words for the IM test as well as 20 new, 20 old and 20 recombined word-pairs in the AM test. There was a self-paced break in between blocks as well as between the two test-lists. After the baseline test, participants were informed about which group they belonged to. At around 17:30 ( \pm 15 minutes) the second test (posttest) was conducted. The posttest consisted of 60 old single and 60 new single words for the IM test as well as 40 new, 40 old and 40 recombined word-pairs in the AM test. The response procedure and test order was the same as in the baseline test and remained constant for each participant.

\subsection{Data acquisition and processing}

\subsubsection{Electroencephalogram (EEG)}

EEG was recorded with BrainVision Recorder Version 1.20 (Brain Products) throughout the entire experiment. In total, $32 \mathrm{Ag} / \mathrm{AgCl}$ electrodes were used according to the 
extended 10-20 system, including electrodes which were located above and below the right eye and outside the outer canthi of both eyes in order to assess electro-ocular activity. Data were recorded with amplifier band pass filter settings from DC to $100 \mathrm{~Hz}$ and a Notch-filter at $50 \mathrm{~Hz}$. The sampling rate was $500 \mathrm{~Hz}$ for all study and test phases. All electrodes were recorded referenced to the left mastoid electrode and re-referenced to the average of the left and right mastoid (offline). Electrode impedances were kept below $5 \mathrm{k} \Omega$. EEG was also recorded at 32 standard locations for polysomnographic data acquisition during the nap; but with a sampling rate of $1000 \mathrm{~Hz}$ and with the inclusion of 2 electrodes at the chin for electromyographic recordings.

\subsubsection{Event-Related Potentials}

Data processing was conducted offline with EEProbe (ANT Software) for ERP analysis of the posttest. A digital $0.2-30 \mathrm{~Hz}$ band-pass filter was first applied. Individual epochs of 1100 ms were then created, including a $100 \mathrm{~ms}$ baseline before stimuli onset. The waveforms were baseline corrected (i.e. the mean value of the baseline was subtracted from each data point in the waveform), before correction of eye-movements and blinks with a linear regression algorithm (Gratton, Coles, \& Donchin, 1983). After this and the rejection of other trials showing artifacts (whenever the standard deviation in a $200 \mathrm{~ms}$ time interval exceeded 25 microvolt at one of the EOG channels), the remaining trials were averaged and individual averages were only used for analyzing ERPs when they contained a minimum of 13 artifact-free trials (Addante, Ranganath, \& Yonelinas, 2012; Gruber \& Otten, 2010). A 12-Hz low pass filter was applied for illustration purposes only.

\subsubsection{Sleep stage scoring}

Preprocessing of the sleep data was conducted using BrainVision Analyzer (2.0, Brain Products). Each $30 \mathrm{sec}$ epoch of sleep was scored visually into rapid-eye-movement (REM)- 
sleep or Non-REM sleep stages 1, 2, 3 or 4 according to standard criteria (Rechtschaffen \& Kales, 1968). Slow-wave-sleep was calculated as the sum of sleep stages 3 and 4 . The time in minutes for each sleep stage, the total sleep time, the sleep onset latency and the percentage of sleep time in each stage with reference to total sleep time (TST) were determined.

\subsubsection{Sleep spindle analysis}

After preprocessing, sleep spindle detection was conducted with MATLAB 2011b (MathWork) for the Fz, $\mathrm{Cz}$ and $\mathrm{Pz}$ recording sites based on an established method (Ferrarelli et al., 2007). In brief, a band-pass filter between 12 and $15 \mathrm{~Hz}$ was applied. Time intervals containing muscle artifacts or analog/digital saturation were excluded. Following this, the envelope of the individual sleep EEG signal was computed using the Hilbert transform and its resulting absolute values. The computed envelope leads to a smoothing of the signal by outlining the extremes in EEG amplitudes. For each participant unique thresholds for spindle detection were used which were the mean plus two SD (lower threshold) and the mean plus four SD (higher threshold) of the participant's filtered EEG signal. To classify a spindle, two criteria had to be fulfilled: i) the duration between the points at which the signal falls above and below the lower threshold needed to be at least $500 \mathrm{~ms}$ and ii) the signal also had to cross the upper threshold within this $500 \mathrm{~ms}$ time window. Spindle density was calculated for NREM (S2+SWS) sleep by dividing the number of spindles by minutes of NREM sleep and for SWS by dividing the number of spindles during SWS by minutes of SWS.

\subsection{Data Analysis}

For the behavioral data, analyses of variance (ANOVA) with factors of group (nap/control) and time (baseline/posttest) were used separately for item memory (IM) and associative memory (AM). For IM tests, an old/new discrimination Pr index (Pr-Score) was 
calculated by subtracting false alarms to new pairs from the hit rate $(\mathrm{Pr}=$ hits-FAneu $)$. In $\mathrm{AM}$ tests, the ability of participants to discriminate between old and recombined pairs was of particular interest, so an associative PrA-Score was computed. This was calculated by subtracting the proportion of recombined pairs which were incorrectly classified as old (false alarms to recombined) from the hit rate $(\operatorname{PrA}=$ hits-FA $\mathrm{Aec})$. By including recombined pairs in the test phases, it was ensured that participants could not make their response based on item memory alone but that they needed to retrieve the associations.

For the reaction time data, ANOVAs with the factors group (nap/control) and the withinsubject factors time (baseline/post) and item-type (IM: old/new; AM: old/new/recombined) were conducted separately for correct answers for IM and AM tests.

ERPs were derived from the posttest EEG data. ERPs in the IM test were limited to correct responses to old (hit) and new (CR) items. For the AM test, recombined items were created such that both items were re-presented at test, albeit with different old items. Recombined pairs were included in the test phase to ensure participants responded on the basis of associative recognition and could not make their responses solely on the basis of item memory. In line with previous ERP studies on associative memory, recombined pairs were not included in the ERP analyses because of difficulties in interpretation, a lack of artifact-free trials (Greve, van Rossum, \& Donaldson, 2007; Kriukova et al., 2013) and because they are not necessary to test our central prediction, namely that nap sleep should increase the amount of recollected information, and that this should be reflected in the amplitude of the late parietal old/new effect (Vilberg et al., 2006). ERP analyses in the AM test were thus restricted to correctly responded to old and new items. To create a subject average, at least 13 artifact-free trials were needed in each of the categories. For the IM test, one participant of the nap group had to be excluded and for the AM test, 3 participants of the nap and 4 participants of the control group needed to be excluded for the ERP analysis but were retained for all other analyses. Mean amplitudes in an early (300-500ms) and a late (500-700 ms) time window were subjected to 
ANOVAs with factors of group (nap/control), item-type (hit/CR) and laterality (left/midline/right). ANOVAs included amplitudes from three frontal (F3, Fz, F4) electrodes for the early time interval and three parietal $(\mathrm{P} 3, \mathrm{Pz}, \mathrm{P} 4)$ electrodes for the late time window. These sites and time points correspond to the standard specifications of the early frontal and late parietal putative correlates of familiarity and recollection (Rugg \& Curran, 2007). Only main effects and interactions that involve the factor item-type are reported because these indicate that an old/new difference is present or varies with group or electrode location. Where necessary, analyses included Greenhouse-Geisser corrections for nonsphericity with corrected p-values and uncorrected degrees of freedom (Greenhouse \& Geisser, 1959). For all analyses, the significance level was set to $\alpha=0.05$ and for the correlation analyses a modified Bonferroni test (Keppel, 1991) was used to correct for multiple comparisons.

\section{RESULTS}

\subsection{Behavioral data}

- Insert Figure 2 around here -

Fig. 2 shows the mean Pr-/PrA-Scores for the IM (a) and AM (b) baseline and posttest for both groups. The hit rates, false alarm rates and the bias index $\mathrm{Br}$ are shown in Table 1. To test the hypothesis that group differences will be present in the AM posttest but not in the IM posttest or IM/AM baseline tests, separate two-way ANOVAs (with factors group and time) were conducted for the Pr (IM Test) and PrA scores (AM test). For IM tests, a main effect of time $(F(1,39)=22.018, \mathrm{p}<.01)$ but no group $\mathrm{x}$ time interaction $(\mathrm{p}=.17)$ was found. For AM tests, the corresponding ANOVA revealed a main effect of time $(F(1,39)=17.176, \mathrm{p}<.01)$ and a significant group $\mathrm{x}$ time interaction $(F(1,39)=9.238, \mathrm{p}<.01)$. PrA scores at posttest were lower than at baseline in the control group $(\mathrm{t}(18)=4.79, \mathrm{p}<.01)$, whereas in the nap group, there was 
no difference between PrA scores at baseline and posttest $(\mathrm{p}=.42)$. At posttest the difference in memory performance between the nap and control group was marginally significant $(\mathrm{p}<.06)$.

Table 1 Hit rates (Hits), false alarm rates (FA) and bias index ( $\mathrm{Br} / \mathrm{BrA})$ for both groups and tests (standard deviation in parentheses) are depicted.

\begin{tabular}{l|l|l|l|l|l|l|l|l|l|l|l|l|l} 
& \multicolumn{5}{c|}{ Item Memory } & \multicolumn{5}{c|}{ Associative Memory } \\
\hline \hline & \multicolumn{5}{|c|}{ Baseline Test } & \multicolumn{3}{c|}{ Posttest } & \multicolumn{3}{c|}{ Baseline Test } & \multicolumn{3}{c}{ Posttest } \\
\hline & Br & Hits & FA & Br & Hits & FA & BrA & Hits & FA & BrA & Hits & FA \\
\hline Nap & 0.34 & 0.71 & 0.15 & 0.37 & 0.66 & 0.19 & 0.06 & 0.61 & 0.17 & 0.10 & 0.55 & 0.14 \\
& $(0.17)$ & $(0.14)$ & $(0.10)$ & $(0.20)$ & $(0.20)$ & $(0.15)$ & $(0.04)$ & $(0.17)$ & $(0.11)$ & $(0.08)$ & $(0.22)$ & $(0.10)$ \\
\hline Control & 0.38 & 0.74 & 0.15 & 0.35 & 0.64 & 0.20 & 0.07 & 0.63 & 0.18 & 0.12 & 0.49 & 0.20 \\
& $(0.26)$ & $(0.16)$ & $(0.11)$ & $(0.10)$ & $(0.12)$ & $(0.11)$ & $(0.05)$ & $(0.21)$ & $(0.13)$ & $(0.05)$ & $(0.19)$ & $(0.09)$
\end{tabular}

To explore whether response bias was modulated by the sleep and wake conditions a two-way ANOVA (factors group and time) was conducted for $\mathrm{Br}$ in both tests. No effects were obtained for the bias index in the IM test (p-values >.30). In the AM test, the bias index increased from baseline to posttest $(F(1,39)=27.28, \mathrm{p}<.001)$, suggesting that participants responded more liberally at posttest irrespective of nap/control condition. Control measures are displayed in Table 2. T-tests revealed no group differences for most of these measures except for SSS3 and SSS4 (Table 2). A mixed ANOVA with time of sleepiness (four levels) and group as factors revealed a main effect of time of sleepiness $(F(3,37)=18.18, \mathrm{p}<.01)$ and a significant interaction between time of sleepiness and group $(F(3,37)=3.40, \mathrm{p}<.05)$. Paired t-tests revealed that both groups reported being more awake at the end of study (after washing their hair) compared to after watching DVD (p-values <.01 in both groups). The interaction reflects the fact that the nap group reported being more awake at SSS3 (after DVD) compared to SSS2 
(after baseline test) $(\mathrm{p}<.01)$ whereas reported sleepiness in the control group did not differ between SSS2 and SSS3 (p=.62). Sleepiness was also reported to be higher in the control group than in the nap group at SSS3 and SSS4 but not at the other two time points. To rule out the possibility that significant group differences in sleepiness before posttest differentially impacted memory performance for the two groups, an ANCOVA with factors group and time and with sleepiness score at SSS3 as a covariate was conducted on PrA-scores. The interaction between group and time remained significant $(\mathrm{p}<.05)$ indicating that differences in sleepiness cannot explain group differences in memory performance.

Table 2 Control measures

\begin{tabular}{l|l|l|l|l} 
& Nap mean (SD) & Control mean (SD) & $\mathbf{t} 39$ & $\mathbf{p}$ \\
\hline IQ (CFT 20-R) & $113.01(12.8)$ & $110.95(12.4)$ & 0.54 & .59 \\
\hline ESS & $7.59(3.53)$ & $7.37(2.99)$ & 0.22 & .83 \\
\hline TST night before experiment & $6.9(1.1)$ & $7.2(0.9)$ & -1.07 & .29 \\
\hline TST average across 7 nights & $7.4(1.2)$ & $7.4(1.1)$ & 0.17 & .87 \\
\hline Wake-up time morning (hh:mm) & $7: 49(1: 06)$ & $8: 10(1: 12)$ & -0.97 & .34 \\
\hline Sleepiness before learning (SSS1) & $2.8(1.2)$ & $2.7(1.2)$ & 0.09 & .93 \\
\hline Sleepiness after baseline test (SSS2) & $3.2(1.2)$ & $3.3(1.1)$ & -0.25 & .80 \\
\hline Sleepiness after DVD (SSS3) & $2.0(0.6)$ & $3.1(1.5)$ & -3.09 & $.01^{*}$ \\
\hline Sleepiness at end of study (SSS4) & $1.6(0.5)$ & $2.1(0.9)$ & -2.42 & $.03^{*}$ \\
\hline
\end{tabular}

A summary of sleep parameters for the nap group is shown in Table 3. The average time spent in sleep was about 64 minutes and about half of this time (51.5\%) was spent in stage 2 (S2) sleep. Participants showed about 24.7\% (SD 18.8) of slow-wave-sleep (SWS) and 8.6\% (SD 9.6) of rapid-eye-movement (REM) sleep. Most participants showed SWS ( $\mathrm{n}=19)$ and nearly 
half of them reached REM sleep (REM: $n=12$ ) which accounts for the large variability of these measures.

Table 3 Sleep parameters

\begin{tabular}{l|l|l} 
& Minutes mean (SD) & \% of TST mean (SD) \\
\hline SL & $14.18(12.53)$ & \\
\hline TST & $64.25(16.3)$ & \\
\hline Stage 1 (S1) & $9.64(7.84)$ & $15.14(10.97)$ \\
\hline Stage 2 (S2) & $32.77(10.85)$ & $51.49(13.13)$ \\
\hline Stage 3 (S3) & $11.2(9.94)$ & $17.13(14.08)$ \\
\hline Stage 4 (S4) & $4.52(5.21)$ & $7.61(9.22)$ \\
\hline SWS (S3+S4) & $15.73(12.19)$ & $24.74(18.78)$ \\
\hline REM & $6.11(6.74)$ & $8.63(9.63)$ \\
SL=latency until sleep onset; TST=total sleep time; SWS=slow-wave-sleep
\end{tabular}

Mean reaction times for each of the conditions are shown for IM and AM for the two groups in Table 4. For IM tests, a three-way ANOVA with factors of group, item-type and time only revealed a main effect of item-type $(F(1,39)=16.412, \mathrm{p}<.01)$ with response times to hits being faster than to correct rejections. For AM, there was also a main effect of item-type $(F(2,38)=42.81, \mathrm{p}<.01)$, again because response times to old items were faster than to new items $(\mathrm{p}<.01)$ and recombined word-pairs $(\mathrm{p}<.01)$ and because reaction times for new pairs were faster compared to recombined pairs $(\mathrm{p}<.01)$. 
Table 4 Mean reaction times [ms] and SD (in parentheses) for all conditions of Item Memory (IM) and Associative Memory (AM) baseline and posttest for the nap and the control group

\begin{tabular}{|c|c|c|c|c|c|c|c|c|c|c|}
\hline & \multicolumn{4}{|c|}{ Item Memory Test } & \multicolumn{6}{|c|}{ Associative Memory Test } \\
\hline & \multicolumn{2}{|c|}{ Baseline Test } & \multicolumn{2}{|c|}{ Posttest } & \multicolumn{3}{|c|}{ Baseline Test } & \multicolumn{3}{|c|}{ Posttest } \\
\hline & Hits & CR & Hits & CR & Hitsold & CR & HitsREC & HitsoLD & CR & HitsReC \\
\hline Nap & $\begin{array}{c}870.93 \\
(209.77)\end{array}$ & $\begin{array}{c}961.90 \\
(284.71)\end{array}$ & $\begin{array}{c}894.65 \\
(197.90)\end{array}$ & $\begin{array}{c}963.72 \\
(257.88)\end{array}$ & $\begin{array}{l}1497.71 \\
(301.82)\end{array}$ & $\begin{array}{l}1622.27 \\
(362.22)\end{array}$ & $\begin{array}{l}1754.15 \\
(329.04)\end{array}$ & $\begin{array}{l}1491.24 \\
(292.01)\end{array}$ & $\begin{array}{l}1576.12 \\
(362.24)\end{array}$ & $\begin{array}{r}1767.08 \\
(321.47)\end{array}$ \\
\hline Control & $\begin{array}{c}838.46 \\
(192.34)\end{array}$ & $\begin{array}{c}896.28 \\
(235.77)\end{array}$ & $\begin{array}{l}822.28 \\
(165.10)\end{array}$ & $\begin{array}{c}869.19 \\
(245.96)\end{array}$ & $\begin{array}{l}1385.64 \\
(253.92)\end{array}$ & $\begin{array}{l}1535.43 \\
(357.20)\end{array}$ & $\begin{array}{l}1684.11 \\
(374.90)\end{array}$ & $\begin{array}{l}1374.21 \\
(259.92)\end{array}$ & $\begin{array}{l}1407.75 \\
(332.35)\end{array}$ & $\begin{array}{l}1580.68 \\
(369.52)\end{array}$ \\
\hline
\end{tabular}

\subsection{Electrophysiological data}

- Insert Figure 3 around here -

\subsubsection{Item Memory Test}

The grand average ERP data for the IM at posttest for both groups are presented in Figure 3. Differences between hits and correct rejections emerge at around $300 \mathrm{~ms}$ at frontal recording sites in the control group and are slightly delayed in the Nap group. Starting at around $500 \mathrm{~ms}$, there are pronounced and posteriorly distributed old/new effects in both groups (Fig. 4). Three-way ANOVAs with factors of group, item-type and lateralization (left/midline/right) performed for both the early time window (at frontal sites) and late time window (at posterior sites) revealed main effects of item-type (early: $F(1,39)=5.23, \mathrm{p}<.05$ and late: $F(1,39)=28.56$, $\mathrm{p}<.01)$. For the late time window, the interaction between item-type and lateralization did not reach significance $(F(2,38)=2.44, \mathrm{p}=.11)$. There were no interactions including the factors itemtype and group.

- Insert Figure 4 around here - 


\subsubsection{Associative Memory Test}

ERPs elicited by correct old and new responses in the AM posttest (Fig. 4) at frontal and parietal recording sites were compared between two sub-groups (nap: $n=19$; control: $n=15$ ). The ERPs shown in Figure 4 indicate that both groups show more positive going waveforms for hits compared to correct rejections at posterior sites from approximately $500 \mathrm{~ms}$ onwards. A three-way ANOVA for the early time window (300-500ms) at frontal sites revealed neither a significant main effect for item-type nor any interaction including the factor item-type (all pvalues $>.43$ ), thus providing no evidence of an early mid-frontal old/new effect in either group. For the late time window (500-700ms) at posterior sites, a marginally significant main effect of item-type was present $(F(1,32)=3.184, \mathrm{p}=.08)$, but again, there was no interaction with the group factor.

To summarize, the ERP analyses for the item memory test revealed an early mid-frontal and a late parietal old/new effect, the putative ERP correlates of familiarity and recollection, respectively. Conversely, only a marginally significant late parietal old/new effect was obtained for the associative memory test and no differences were obtained between the two groups in either memory test.

\subsection{Sleep spindle data}

A correlation between spindle density $(\mathrm{SpD})$ and PrA score at AM posttest but not between spindle density and Pr-score at IM posttest was expected. In line with other reports of spindle density analyses, data from 3 midline electrodes $-\mathrm{Fz}, \mathrm{Cz}$ and $\mathrm{Pz}$ - were examined (Gais et al., 2002; Saletin et al., 2011) for the total amount of NREM sleep as well as separately for SWS (Cox et al., 2012). There were no significant correlations between spindle density in NREM sleep and Pr at IM posttest (all p-values >.5) or between spindle density in NREM sleep and PrA at AM posttest (all p-values >.1). Notably, an analysis of the subset of participants 
$(n=19)$ who did reach SWS revealed a significant correlation between PrA at AM posttest and spindle density in SWS at Fz $(r=0.59 ; \mathrm{p}<.01$, Figure 5a) that remained significant when correcting for multiple testing.

To explore whether the latter correlation between spindle density and AM posttest performance is modulated by performance at baseline, we conducted a partial correlation analysis between posttest memory performance and spindle density with memory performance at baseline as covariate. The correlation is no longer significant when the baseline performance is controlled for, which is not surprising given the high common variance between AM baseline and posttest performance $(\mathrm{r}=.0 .76, \mathrm{p}<.001)$.

- Insert Figure 5 around here -

In some reports, spindle density has been shown to correlate with memory performance/learning prior to sleep (Gais et al., 2002; Schmidt et al., 2006). To explore this possibility in the current data set, we conducted correlation analyses between baseline performance measures and spindle density at $\mathrm{Fz}, \mathrm{Cz}$ and $\mathrm{Pz}$. There was no correlation between the Pr-score of the baseline IM test and spindle density in NREM sleep (all p-values>.271) but the correlation between the PrA score at AM baseline test and spindle density in NREM sleep at $\mathrm{Fz}$ was marginally significant $(\mathrm{r}=0.45 ; \mathrm{p}=.036$; Fig. $5 \mathrm{~b})$ when corrected for multiple comparisons $^{2}$.

\footnotetext{
${ }^{2}$ The adjusted significance level used to evaluate the correlations between spindle density at the three electrodes in the modified Bonferroni test was p=.034 (Keppel, 1991).
} 
To summarize the results of the correlation analyses, positive relationships between PrA scores at AM posttest and spindle density during SWS as well as between PrA score at baseline test and spindle density during NREM sleep were found in the AM task only.

\section{DISCUSSION}

An associative memory task was compared with an item memory task to explore the effects of nap sleep on different forms of recognition memory. It was predicted that a memory benefit for the nap group relative to the wake control group would be observed only for recollection-dependent measures in the AM test - in this case, PrA scores (differentiation between old and recombined pairs) - after the retention period (AM posttest) whereas no group differences for Pr-scores in the IM task (old/new differentiation) should arise. In line with these predictions for the IM test, no group differences in behavioral measures of recognition memory were observed at baseline or posttest, and both groups showed a decrease in performance from baseline to posttest. As predicted, a different picture emerged for the AM test. While the control group showed a significant deterioration from AM baseline to AM posttest, performance in the nap group remained constant over time. This finding is consistent with studies showing that short periods of sleep are sufficient to induce a measurable benefit in declarative memory (Cox et al., 2012; Saletin et al., 2011; Schmidt et al., 2006; Tucker et al., 2006). It also adds to the few recognition memory studies which show a beneficial impact of sleep for recollection but not for familiarity (Daurat et al., 2007; Drosopoulos et al., 2005)

In addition to findings of other studies in which benefits of sleep on recognition memory were reported, we found a selective correlation between AM posttest performance and spindle density during SWS. This is in accordance with recent evidence that memory consolidation processes rely on sleep spindles and co-occurring slow oscillations during SWS (Cox et al., 2012). The role of SWS for memory consolidation was also revealed in a recent stimulation 
study in which slow oscillatory activity was enhanced via auditory stimulation. Stimulation in phase with ongoing rhythmic slow oscillations enhances grouping of slow oscillations and phase coupled spindle activity and in turn improved declarative memory (Ngo, Martinetz, Born, \& Molle, 2013). In fact, spindle activity and percentage of SWS showed a strong positive correlation with the overnight retention of word-pairs. The authors concluded that it is the synchronization of spindles with slow oscillations which might be critical for memory consolidation (Ngo et al., 2013). The correlation between AM posttest performance and spindle density in SWS in the present study may thus provide further evidence for the active system consolidation hypothesis (Born \& Wilhelm, 2012).

The current data also show that AM baseline performance before the nap correlated with spindle density in the following sleep period such that controlling for baseline performance removed the relationship between posttest memory performance and spindle density. One reason for this is because of the general association between baseline and posttest memory performance. It is also possible that the current correlations reflect the possibility that baseline performance has an impact on both spindle density and posttest memory performance. Indeed a number of studies have also reported relationships between sleep parameters such as spindle density and memory performance prior to the sleep period (Gais et al., 2002; Schmidt et al., 2006). Gais and colleagues (2002), for example, found positive correlations between spindle density at fronto-central sites and cued recall performance in a declarative paired-associate task both before and after a night of sleep but not in a non-learning task which was matched in all stimulus and task characteristics except the intention to learn. This pattern is in line with the possibility that these spindles relate to intentional learning and speaks for the presence of a common mechanism involved in sleep spindle generation and intentional build-up of long-term memory representations (Gais et al., 2002). The present study supports this possibility by demonstrating a positive correlation between spindle density at Fz and memory performance in the AM baseline test. Another study conducted by Schmidt and colleagues (2006) reported a 
relationship between pre-sleep encoding difficulty and spindle density. Here, participants learned lists of unrelated word-pairs in two different conditions. One condition comprised concrete words that could easily be encoded on the basis of preexisting semantic knowledge, whereas the abstract words employed in the second condition were (assumed to be) more difficult to encode. Spindle density was significantly increased over left frontal cortex for difficult but not for easy to encode word-pairs and spindle density was positively correlated with nap-related changes in memory performance. This finding is consistent with the view that sleep modulates memory consolidation when completely new memory associations are built up (as is presumed to be the case for the difficult to encode pairs) and less so when encoding relies on pre-existing semantic relations (Schmidt et al., 2006). Consistent with the two aforementioned studies, the correlation between AM baseline memory performance and spindle density in NREM sleep in the current data set can be taken as further evidence that sleep only consolidates associative memories which are efficiently built up in the pre-sleep period as reflected in superior AM performance.

Another prediction derived from the current design was that the late parietal old/new effect - the putative ERP correlate of recollection - should be larger after nap sleep compared to the control group whereas no corresponding differences for the early mid-frontal old/new effect were expected. There was evidence of a late parietal old/new effect as well as an early frontal old/new effect in the IM test in both groups in accordance with the assumption that successful performance in the item memory task is associated with both familiarity and recollection. Neither of these old/new effects was modulated by sleep, however. Comparable early mid-frontal old/new effects in both groups supports the view that item memory for which no contextual information is provided is not modulated by sleep (Drosopoulos et al., 2005; Hu et al., 2006). However, as recollection also occurs for item memory a group difference in the amplitude of the late parietal old/new effect was also expected, but this was not observed. 
For the AM posttest data, there was no observable early frontal old/new effect, in line with the assumption that familiarity does not contribute to associative tests with arbitrary associations (Yonelinas et al., 2010). The late parietal old/new effect was marginally significant in line with the notion that recollection is required for this task. The amplitude of the effect in this task also did not differ between nap and wake groups, however, and the behavioral interaction for the AM test (less forgetting in AM after nap sleep) was not paralleled by corresponding changes in the ERP correlates of recollection. There are several possible reasons for the discrepancy between behavioral and ERP measures of recognition memory. One possibility could be that the late parietal old/new effect is not sensitive enough to detect subtle changes in recollective processing and the retrieval of contextual information set in train by the prior nap period. Although this effect has been shown to be sensitive to the amount of information recollected, when manipulated experimentally within subjects (Vilberg et al., 2006), it might be the case that the differences in recollection that arise from the preceding wake vs. nap between-subject manipulation are not large enough to be reflected in group differences in the late parietal old/new effect. Another possibility is that increases in the amount of recollected information are not the underlying basis of the beneficial effect of sleep over wake. According to this view, beneficial effects of sleep in recognition memory studies might not necessarily manifest as an increase in the ERP correlate of recollection. Rather it could come about because of enhanced flexibility of retrieval processing and/or facilitated access to associative memories and the discrimination between old and rearranged word pairs without being reflected in amplitude differences in the late parietal old/new effect. The suggestions put forward here do not necessarily oppose one another and it is conceivable that sleep boosts associative memory via a variety of mechanisms. Taken together our findings raise the question how the left parietal old/new effect reflects variance in recollection across subjects and further work is required to explore this relationship in more detail. Notably however, our findings also suggest that the comparison of two recognition memory tasks from which only one requires 
recollective processing is a more powerful way to disentangle the differential effects of nap sleep on familiarity and recollection.

To conclude, the present study showed a differential influence of nap sleep compared to a wake retention period on associative memory but no corresponding effects for item memory. The selective effect of nap sleep at AM posttest memory performance is consistent with the view that even short periods of nap sleep have a beneficial effect on Hc-dependent memory consolidation. The beneficial effects of nap sleep on post-sleep AM performance were not paralleled by differences in the ERP correlate of recollection, suggesting either that the latter effect is not sufficiently sensitive to capture small between-group differences in recollective processing or that sleep boosts associative memory by improving the flexibility of retrieval processing more generally. Positive correlations between spindle density in SWS and AM postsleep performance and between spindle density in NREM sleep and AM baseline test performance were found. The former effect adds to the increasing evidence that SWS is of high relevance for the consolidation of declarative memories although the possibility that baseline performance determines both spindle density and posttest memory performance cannot be excluded from the current data. On the basis of the correlation between spindle density in NREM sleep and AM baseline performance it is tempting to speculate that sleep only consolidates memories which are efficiently built up and newly formed prior to the sleep period (Stickgold \& Walker, 2013) and further studies will be required to unravel by which mechanisms the brain distinguishes between information that is retained or forgotten by sleep.

\section{Acknowledgements}

We gratefully acknowledge Anna Borchert, Ruta Garbacenkaite, Stephanie Raschka and Ruohan Li for assisting with data collection/analysis and Sebastian Hinrichsen for the 
development of the spindle detection algorithm. This research was supported by a grant from the German Research Foundation (IRTG 1457).

\section{References}

Addante, R. J., Ranganath, C., \& Yonelinas, A. P. (2012). Examining ERP correlates of recognition memory: Evidence of accurate source recognition without recollection. Neuroimage, 62(1), 439-450. doi: 10.1016/j.neuroimage.2012.04.031

Baayen, R. H., Piepenbrock, R., \& Gulikers, L. (1995). The CELEX lexical database [Webcelex]. Philadelphia, PA, USA: University of Pennsylvania, Linguistic Data Consortium.

Bader, R., Mecklinger, A., Hoppstadter, M., \& Meyer, P. (2010). Recognition memory for one-trialunitized word pairs: Evidence from event-related potentials. Neuroimage, 50(2), 772-781. doi: 10.1016/j.neuroimage.2009.12.100

Born, J., Rasch, B., \& Gais, S. (2006). Sleep to remember. Neuroscientist, 12(5), 410-424. doi: $10.1177 / 1073858406292647$

Born, J., \& Wilhelm, I. (2012). System consolidation of memory during sleep. Psychological ResearchPsychologische Forschung, 76(2), 192-203. doi: 10.1007/s00426-011-0335-6

Bridger, E. K., Bader, R., \& Mecklinger, A. (2014). More ways than one: ERPs reveal multiple familiarity signals in the word frequency mirror effect. Neuropsychologia, 57, 179-190. doi: 10.1016/j.neuropsychologia.2014.03.007

Bridger, E. K., \& Mecklinger, A. (2012). Electrophysiologically Dissociating Episodic Preretrieval Processing. Journal of Cognitive Neuroscience, 24(6), 1476-1491.

Cox, R., Hofman, W. F., \& Talamini, L. M. (2012). Involvement of spindles in memory consolidation is slow wave sleep-specific. Learning \& Memory, 19(7), 264-267. doi: 10.1101/Im.026252.112

Curran, T., \& Cleary, A. M. (2003). Using ERPs to dissociate recollection from familiarity in picture recognition. Cognitive Brain Research, 15(2), 191-205. doi: 10.1016/s0926-6410(02)00192-1

Daurat, A., Terrier, P., Foret, J., \& Tiberge, M. (2007). Slow wave sleep and recollection in recognition memory. Consciousness and Cognition, 16(2), 445-455. doi: 10.1016/j.concog.2006.06.011

Diekelmann, S., \& Born, J. (2010). SLEEP The memory function of sleep. Nature Reviews Neuroscience, 11(2), 114-126. doi: 10.1038/nrn2762

Diekelmann, S., Wilhelm, I., \& Born, J. (2009). The whats and whens of sleep-dependent memory consolidation. Sleep Medicine Reviews, 13(5), 309-321. doi: 10.1016/j.smrv.2008.08.002

Drosopoulos, S., Wagner, U., \& Born, J. (2005). Sleep enhances explicit recollection in recognition memory. Learning \& Memory, 12(1), 44-51. doi: 10.1101/kfg.mu-luebeck.de

Ferrarelli, F., Huber, R., Peterson, M. J., Massimini, M., Murphy, M., Riedner, B. A., . . Tononi, G. (2007). Reduced sleep spindle activity in schizophrenia patients. Am J Psychiatry, 164(3), 483492. doi: 10.1176/appi.ajp.164.3.483

Friedman, D., \& Johnson, R. (2000). Event-related potential (ERP) studies of memory encoding and retrieval: a selective review. Microsc Res Tech, 51(1), 6-28. doi: 10.1002/10970029(20001001)51:1<6::AID-JEMT2>3.0.CO;2-R

Gais, S., Molle, M., Helms, K., \& Born, J. (2002). Learning-dependent increases in sleep spindle density. Journal of Neuroscience, 22(15), 6830-6834.

Gratton, G., Coles, M. G., \& Donchin, E. (1983). A new method for off-line removal of ocular artifact. Electroencephalogr Clin Neurophysiol, 55(4), 468-484.

Greenhouse, S. W., \& Geisser, S. (1959). ON METHODS IN THE ANALYSIS OF PROFILE DATA. Psychometrika, 24(2), 95-112. doi: 10.1007/bf02289823

Greve, A., van Rossum, M. C., \& Donaldson, D. I. (2007). Investigating the functional interaction between semantic and episodic memory: convergent behavioral and electrophysiological 
evidence for the role of familiarity. Neuroimage, 34(2), 801-814. doi:

10.1016/j.neuroimage.2006.07.043

Gruber, M. J., \& Otten, L. J. (2010). Voluntary Control over Prestimulus Activity Related to Encoding. Journal of Neuroscience, 30(29), 9793-9800. doi: 10.1523/jneurosci.0915-10.2010

Hockley, W. E., \& Consoli, A. (1999). Familiarity and recollection in item and associative recognition. Memory \& Cognition, 27(4), 657-664. doi: 10.3758/bf03211559

Hu, P., Stylos-Allan, M., \& Walker, M. P. (2006). Sleep facilitates consolidation of emotional declarative memory. Psychological Science, 17(10), 891-898. doi: 10.1111/j.14679280.2006.01799.x

Ji, D., \& Wilson, M. A. (2007). Coordinated memory replay in the visual cortex and hippocampus during sleep. Nat Neurosci, 10(1), 100-107. doi: 10.1038/nn1825

Keppel, G. (1991). Design and analysis: A researcher's handbook (3rd ed.). Englewood Cliffs, NJ, US: Prentice-Hall, Inc.

Kriukova, O., Bridger, E., \& Mecklinger, A. (2013). Semantic relations differentially impact associative recognition memory: Electrophysiological evidence. Brain and Cognition, 83(1), 93-103. doi: 10.1016/j.bandc.2013.07.006

Marshall, L., Molle, M., Hallschmid, M., \& Born, J. (2004). Transcranial direct current stimulation during sleep improves declarative memory. Journal of Neuroscience, 24(44), 9985-9992. doi: 10.1523/jneurosci.2725-04.2004

Mecklinger, A. (2000). Interfacing mind and brain: A neurocognitive model of recognition memory. Psychophysiology, 37(5), 565-582. doi: 10.1111/1469-8986.3750565

Mednick, S. C., McDevitt, E. A., Walsh, J. K., Wamsley, E., Paulus, M., Kanady, J. C., \& Drummond, S. P. A. (2013). The Critical Role of Sleep Spindles in Hippocampal-Dependent Memory: A Pharmacology Study. Journal of Neuroscience, 33(10), 4494-4504. doi: 10.1523/jneurosci.3127-12.2013

Mograss, M., Godbout, R., \& Guillem, F. (2006). The ERP old-new effect: A useful indicator in studying the effects of sleep on memory retrieval processes. Sleep, 29(11), 1491-1500.

Mograss, M. A., Guillem, F., \& Godbout, R. (2008). Event-related potentials differentiates the processes involved in the effects of sleep on recognition memory. Psychophysiology, 45(3), 420-434. doi: 10.1111/j.1469-8986.2007.00643.x

Ngo, H. V. V., Martinetz, T., Born, J., \& Molle, M. (2013). Auditory Closed-Loop Stimulation of the Sleep Slow Oscillation Enhances Memory. Neuron, 78(3), 545-553. doi: 10.1016/j.neuron.2013.03.006

O'Reilly, R. C., Bhattacharyya, R., Howard, M. D., \& Ketz, N. (2011). Complementary Learning Systems. Cogn Sci. doi: 10.1111/j.1551-6709.2011.01214.x

Oldfield, R. C. (1971). THE ASSESSMENT AND ANALYSIS OF HANDEDNESS: THE EDINBURGH INVENTORY. Neuropsychologia, 9(1), 97-113. doi: 10.1016/0028-3932(71)90067-4

Peigneux, P., Laureys, S., Fuchs, S., Collette, F., Perrin, F., Reggers, J., . . Maquet, P. (2004). Are spatial memories strengthened in the human hippocampus during slow wave sleep? Neuron, 44(3), 535-545. doi: 10.1016/j.neuron.2004.10.007

Plihal, W., \& Born, J. (1999). Effects of early and late nocturnal sleep on priming and spatial memory. Psychophysiology, 36(5), 571-582. doi: 10.1017/s0048577299971536

Rasch, B., \& Born, J. (2013). About sleep's role in memory. Physiol Rev, 93(2), 681-766. doi: 10.1152/physrev.00032.2012

Rasch, B., Buechel, C., Gais, S., \& Born, J. (2007). Odor cues during slow-wave sleep prompt declarative memory consolidation. Science, 315(5817), 1426-1429. doi: 10.1126/science.1138581

Rechtschaffen, A., \& Kales, A. (1968). A manual of standardized terminology, techniques and scoring system for sleep stages of human subjects.

Rugg, M. D., \& Curran, T. (2007). Event-related potentials and recognition memory. Trends in Cognitive Sciences, 11(6), 251-257. doi: 10.1016/j.tics.2007.04.004 
Saletin, J. M., Goldstein, A. N., \& Walker, M. P. (2011). The Role of Sleep in Directed Forgetting and Remembering of Human Memories. Cerebral Cortex, 21(11), 2534-2541. doi: 10.1093/cercor/bhr034

Schabus, M., Gruber, G., Parapatics, S., Sauter, C., Klosch, G., Anderer, P., . . Zeitlhofer, J. (2004). Sleep spindles and their significance for declarative memory consolidation. Sleep, 27(8), 1479-1485.

Schmidt, C., Peigneux, P., Muto, V., Schenkel, M., Knoblauch, V., Munch, M., ... Cajochen, C. (2006). Encoding difficulty promotes postlearning changes in sleep spindle activity during napping. Journal of Neuroscience, 26(35), 8976-8982. doi: 10.1523/jneurosci.2464-06.2006

Sirota, A., Csicsvari, J., Buhl, D., \& Buzsaki, G. (2003). Communication between neocortex and hippocampus during sleep in rodents. Proceedings of the National Academy of Sciences of the United States of America, 100(4), 2065-2069. doi: 10.1073/pnas.0437938100

Skinner, E. I., Manios, M., Fugelsang, J., \& Fernandes, M. A. (2014). Reinstatement of encoding context during recollection: behavioural and neuroimaging evidence of a double dissociation. Behav Brain Res, 264, 51-63. doi: 10.1016/j.bbr.2014.01.033

Stickgold, R., \& Walker, M. P. (2013). Sleep-dependent memory triage: evolving generalization through selective processing. Nat Neurosci, 16(2), 139-145. doi: 10.1038/nn.3303

Tucker, M. A., \& Fishbein, W. (2008). Enhancement of declarative memory performance following a daytime nap is contingent on strength of initial task acquisition. Sleep, 31(2), 197-203.

Tucker, M. A., Hirota, Y., Wamsley, E. J., Lau, H., Chaklader, A., \& Fishbein, W. (2006). A daytime nap containing solely non-REM sleep enhances declarative but not procedural memory. Neurobiology of Learning and Memory, 86(2), 241-247. doi: 10.1016/j.nlm.2006.03.005

Vilberg, K. L., Moosavi, R. F., \& Rugg, M. D. (2006). The relationship between electrophysiological correlates of recollection and amount of information retrieved. Brain Res, 1122(1), 161-170. doi: 10.1016/j.brainres.2006.09.023

Wagner, U., Kashyap, N., Diekelmann, S., \& Born, J. (2007). The impact of post-learning sleep vs. wakefulness on recognition memory for faces with different facial expressions. Neurobiology of Learning and Memory, 87(4), 679-687. doi: 10.1016/j.nlm.2007.01.004

Yonelinas, A. P. (1997). Recognition memory ROCs for item and associative information: The contribution of recollection and familiarity. Memory \& Cognition, 25(6), 747-763. doi: $10.3758 /$ bf03211318

Yonelinas, A. P. (2002). The nature of recollection and familiarity: A review of 30 years of research. Journal of Memory and Language, 46(3), 441-517. doi: 10.1006/jmla.2002.2864

Yonelinas, A. P., Aly, M., Wang, W. C., \& Koen, J. D. (2010). Recollection and Familiarity: Examining Controversial Assumptions and New Directions. Hippocampus, 20(11), 1178-1194. doi: 10.1002/hipo.20864

Yonelinas, A. P., Otten, L. J., Shaw, K. N., \& Rugg, M. D. (2005). Separating the brain regions involved in recollection and familiarity in recognition memory. J Neurosci, 25(11), 3002-3008. doi: 10.1523/JNEUROSCI.5295-04.2005 


\section{Figure Legends}

Fig. 1 Study design: Overview and timeline of the experimental procedure on session one (day 1) and session two (day 2). The study phase consisted of 120 word-pairs and 90 single words to be learnt. For the baseline test 60 word-pairs (20 in each category) and 60 single words (30 in each category) were tested. The posttest was double the size of the baseline test.

Fig. 2 Behavioral performance measurement for (a) the item memory test depicting Pr-Scores (hits minus false alarms) and (b) associative memory tests depicting PrA-Scores (hits to old pairs minus false alarms to recombined pairs). Error bars reflect standard deviation. $(* \mathrm{p}<.05)$

Fig. 3 Grand average ERPs elicited by hits and correct rejections at F3, Fz and F4 and P3, Pz and P4 in the item memory posttest for the nap and control group. The arrows highlight the early midfrontal old/new effect and the late parietal old/new effects. The $y$-axis denotes the onset of the test word and negative polarity is plotted upwards.

Fig. 4 Grand average ERPs elicited by hits and correct rejections at electrode positions F3, Fz and F4 and P3, Pz and P4 in the associative memory test at posttest for the nap and control group. The arrow highlights the late parietal old/new effect. The y-axis denotes the onset of the test word pair and negative polarity is plotted upwards.

Fig. 5 Correlation data. (a) Relationship between PrA (hits to old pairs minus false alarms to recombined pairs) scores in the associative memory test at posttest and spindle density per minute at electrode Fz during slow-wave-sleep (SWS). (b) Relationship between PrA scores in the associative memory test at baseline and spindle density per minute at electrode Fz during non-REM sleep (NREM). 
Figure 1

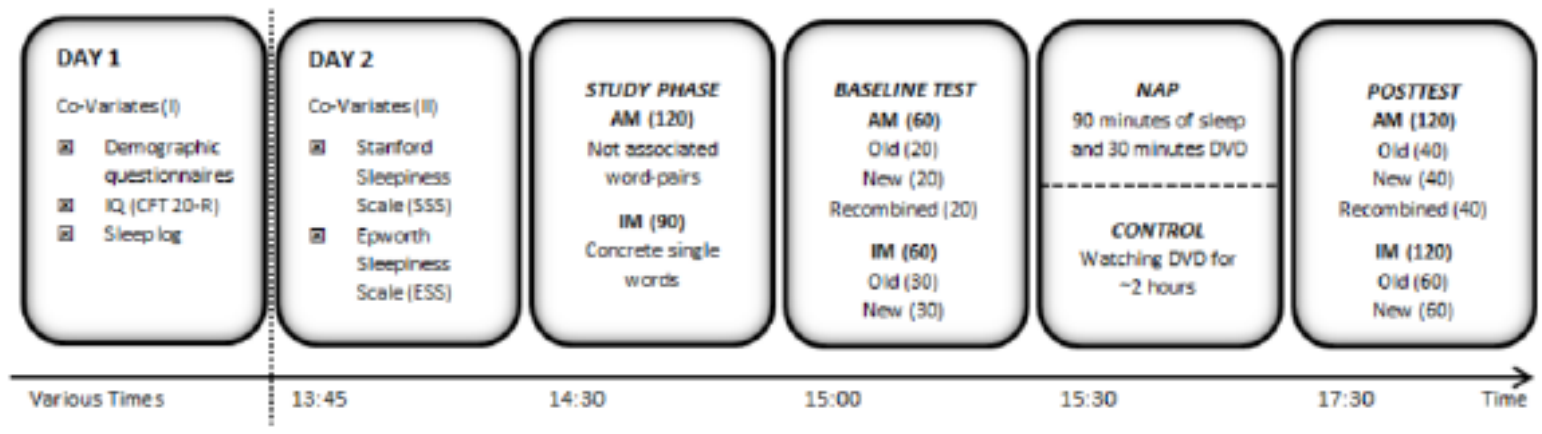

Figure 2

a

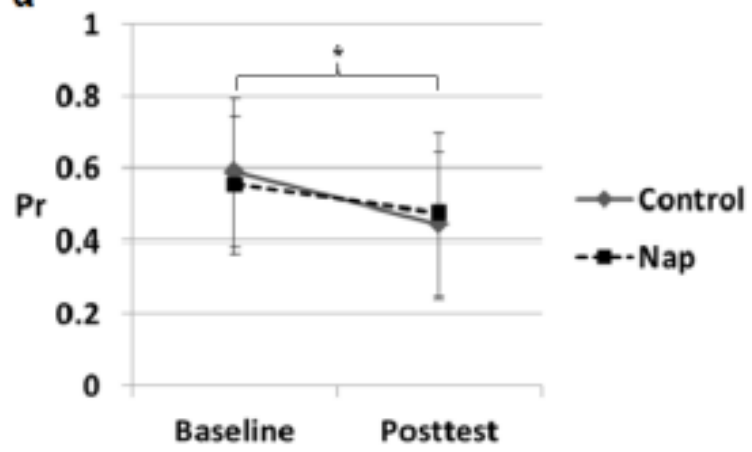

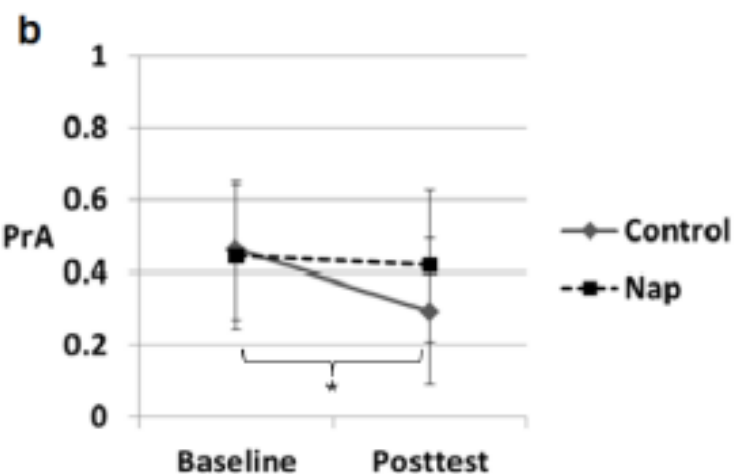

Figure

NAP

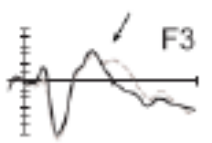

垟A
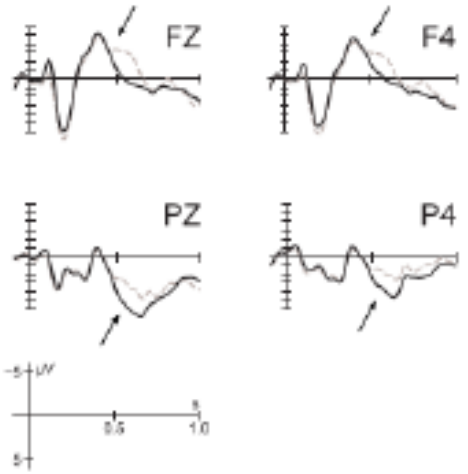

CONTROL
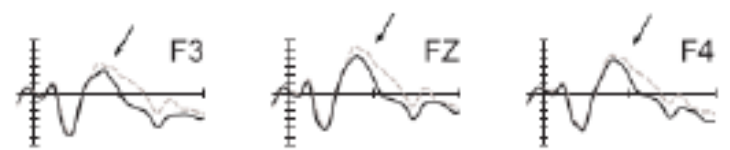

复

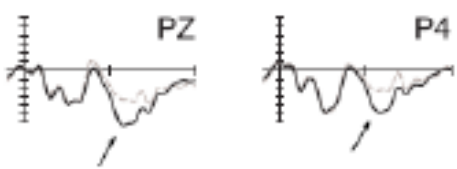

Legend:

-..- Correct Rejections 
Figure 4

NAP

看
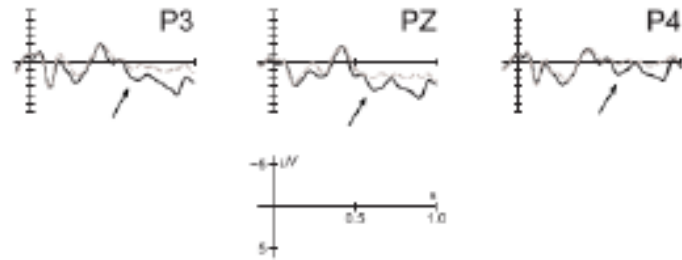

CONTROL

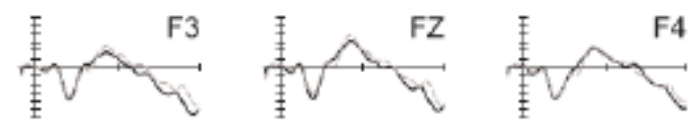

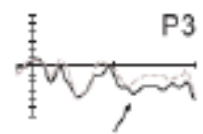

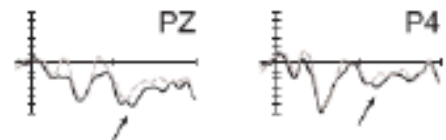

Legend:

Correct Rejoctions

Figure 5
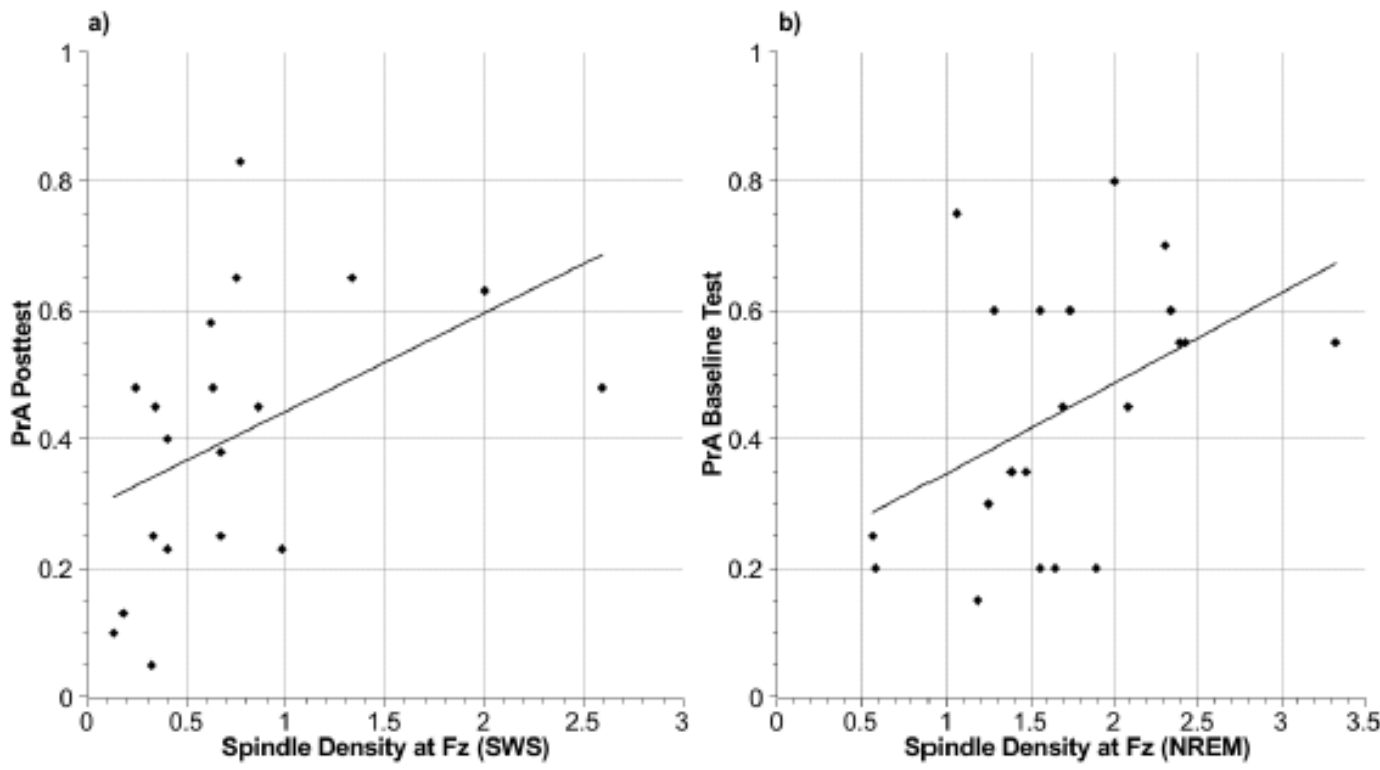